Pacific Journal of Mathematics

RESOLUTION OF SIGN AMBIGUITIES IN JACOBI AND 


\title{
RESOLUTION OF SIGN AMBIGUITIES IN JACOBI AND JACOBSTHAL SUMS
}

\author{
RONALD J. Evans
}

Let $p$ be a prime $\equiv 1(\bmod 16)$. We obtain extensions of known congruences involving parameters of bioctic Jacobi sums $(\bmod p)$. These extensions are used to give an elementary proof of an important congruence of Hasse relating parameters of quartic and octic Jacobi sums $(\bmod p)$. This proof leads directly to an elementary resolution of sign ambiguities of parameters of certain quartic, octic, and bioctic Jacobi and Jacobsthal sums. E. Lehmer's work on ambiguities in quartic sums is thereby extended.

1. Introduction and notation. Throughout this paper, $p=$ $16 f+1$ is a prime with fixed primitive root $g$. Fix a character $\chi(\bmod p)$ of order 16 such that $\chi(g)=\beta$, where $\beta=e^{2 \pi i / 16}$. Let $m=\operatorname{ind}_{g} 2$, so that $2 \equiv g^{m}(\bmod p)$.

For characters $\lambda, \gamma^{\prime}(\bmod p)$, define the Jacobi sums

$$
J\left(\lambda, \psi^{\prime}\right)=\sum_{n(\bmod p)} \lambda(n) \psi(1-n)
$$

and

$$
K(\lambda)=\lambda(4) J(\lambda, \lambda) .
$$

For $\alpha \not \equiv 0(\bmod p)$, define the Jacobsthal sums

$$
\varphi_{n}(\alpha)=\sum_{\nu(\bmod p)}\left(\frac{\nu}{p}\right)\left(\frac{\nu^{n}+\alpha}{p}\right),
$$

where the factors in the summands are Legendre symbols.

It is well known [8, Lemma 2] that if the character $\lambda$ has order $2 n$, then

$$
K(\lambda)=J\left(\lambda, \lambda^{n}\right) .
$$

Simple consequences of this formula are the following (see [1, Theorems 2.5 and 2.7]):

$$
\varphi_{n}(\alpha)=\lambda(-1) \sum_{j=0}^{n-1} \lambda^{n+1+2 j}(\alpha) K\left(\lambda^{2 j+1}\right),
$$

and

$$
K(\lambda)=\lambda(-1) J\left(\lambda, \lambda^{n-1}\right),
$$

where the character $\lambda$ has order $2 n$. 
Using this formula for $K(\lambda)$ and [11, eqs. (3.4), (3.6), (3.10), (3.13)], we see that

$$
\begin{gathered}
K\left(\chi^{4}\right)=-x+2 i y \\
K\left(\chi^{2}\right)=-a+i b \sqrt{2} \\
K(\chi)=c_{0}+c_{2} \sqrt{2}+i c_{1} \sqrt{2-\sqrt{2}}+i c_{3} \sqrt{2+\sqrt{2}}
\end{gathered}
$$

and

$$
J\left(\chi, \chi^{2}\right)=\sum_{i=0}^{7} d_{i} \beta^{i} .
$$

The integers $x$ and $|y|$ are uniquely determined by the conditions

$$
p=x^{2}+4 y^{2}, \quad x \equiv 1(\bmod 4),
$$

and the integers $a$ and $|b|$ are uniquely determined by the conditions

$$
p=a^{2}+2 b^{2}, \quad a \equiv 1(\bmod 4) .
$$

By [2, Theorem 3.5], $c_{0},\left|c_{2}\right|$, and $\left\{\left|c_{1}\right|,\left|c_{3}\right|\right\}$ are uniquely determined by the conditions

$$
\begin{gathered}
p=c_{0}^{2}+2 c_{1}^{2}+2 c_{2}^{2}+2 c_{3}^{2}, \quad c_{0} \equiv-1(\bmod 8), \\
2 c_{0} c_{2}=c_{1}^{2}-c_{3}^{2}-2 c_{1} c_{3} .
\end{gathered}
$$

See also [4, p. 338] and [9]. No simple criteria in terms of $m$ are known to determine in general the signs of the ambiguous parameters in (2), (3), (4).

In $\S 2$, we extend the congruence in (8) by characterizing $c_{0}(\bmod 16)$. This result is then used to give an elementary proof of the congruence

$$
y \equiv 2 b+m(\bmod 16) .
$$

Hasse [5, p. 232] gave a proof of (9) using deep results from class field theory. Also in $\S 2$, we characterize $c_{2}(\bmod 8)$, thus extending the known congruence $c_{2} \equiv m(\bmod 4)$ [2, Theorem 3.6]. The congruences proved in $\S 2$ are used in $\S 3$, for certain $m$, to resolve sign ambiguities in the quartic, octic, and bioctic Jacobi sums in (2), (3), and (4), and in the corresponding Jacobsthal sums $\varphi_{2}(\alpha), \varphi_{4}(\alpha)$, and $\varphi_{8}(\alpha)$.

The cyclotomic number $(i, j)$ of order 16 is defined to be the number of integers $n(\bmod p)$ for which both $n / g^{i}$ and $(1+n) / g^{j}$ 
are 16 th power residues. In [3], the numbers $256(i, j)$ are expressed as linear combinations of the parameters in $(2)-(5)$. We will frequently use these formulas. The idea to apply the cyclotomic numbers of order 16 to resolve sign ambiguities originated in [7, p. 110].

2. Congruences for parameters of Jacobi sums. We record and then justify the following congruences:

$$
\begin{gathered}
y \equiv-m(\bmod 8) ; \\
c_{2} \equiv m \equiv b(\bmod 4) ; \\
a \equiv x-4 b(\bmod 32), \text { when } 4 \mid m ; \\
a \equiv\left\{\begin{array}{l}
(3-p) / 2(\bmod 16), \text { if } 4 \mid m \\
(p-23) / 2(\bmod 32), \text { if } 2 \| m ;
\end{array}\right. \\
x \equiv\left\{\begin{array}{l}
(5-3 p) / 2(\bmod 64), \text { if } 8 \mid m \\
(-3 p-59) / 2(\bmod 64), \text { if } 4 \| m \\
(53-3 p) / 2(\bmod 64), \text { if } 2 \| m .
\end{array}\right.
\end{gathered}
$$

When $2 \| m,(10)$ follows from [7, p. 108, eq. (32)], and when $4 \mid m$, (10) is a special case of [1, Theorem 3.17]. The first congruence in (11) follows from [2, Theorem 3.6] and the second follows from [1, Theorem 3.15]. By (6), (7), and $(10), x^{2} \equiv a^{2}+2 b^{2}(\bmod 64)$ when $4 \mid \mathrm{m}$. Thus (12) follows, with the aid of (11). Finally, (13) and (14) are special cases of [1, Theorem 3.14] and [1, Theorem 3.16], respectively.

Theorem 1 below extends (14).

THEOREM 1. We have

$$
x \equiv \begin{cases}(p+1) / 2(\bmod 128) & \text { if } 2|f, 8| m \\ (p-63) / 2(\bmod 128) & \text { if } 2 \nmid f, 8 \mid m \\ (9 p-71) / 2(\bmod 256) & \text { if } 2 \mid f, 4 \| m \\ (p-127) / 2(\bmod 256) & \text { if } 2 \nmid f, 4 \| m \\ -8 y+(p+145) / 2(\bmod 128), & \text { if } 2 \mid f, 2 \| m \\ 8 y+(p+17) / 2(\bmod 128), & \text { if } 2 \nmid f, 2 \| m .\end{cases}
$$

Proof. The result can be deduced using (6) and (10). For example, in the case $2 \mid f, 4 \| m$, we have $4 y^{2} \equiv 64(\bmod 512)$ by $(10)$. Hence by $(6) x^{2} \equiv p-64(\bmod 512)$ and the result follows.

In the next theorem, we characterize $c_{0}(\bmod 16)$, thus extend- 
ing (8). Recall from (11) that $b \equiv m(\bmod 4)$.

THEOREM 2. We have

$$
c_{0} \equiv\left\{\begin{aligned}
-1(\bmod 16), & \text { if }(8|m, 8| b) \text { or }(4\|m, 4\| b) \text { or }(2 \nmid f, 2 \| m) \\
7(\bmod 16), & \text { if }(8 \mid m, 4 \| b) \text { or }(4 \| m, 8 \mid b) \text { or }(2 \mid f, 2 \| m) .
\end{aligned}\right.
$$

Proof. We shall use the formulas for $256(i, j)$ found in [3]. First suppose that $8 \mid \mathrm{m}$. We must show that

$$
c_{0} \equiv 2 b-1(\bmod 16) \text {. }
$$

Assume that $2 \mid f$. Then

$$
2 p+2+4 x+8 a+16 c_{0}=256\{(1,2)+(3,6)\} \equiv 0(\bmod 256) .
$$

By (12), $8 a$ can be replaced by $8 x-32 b$ above. Then (15) follows with use of (14). Now assume that $2 \nmid f$. Then

$$
\begin{gathered}
2 p-30+4 x-64 y+8 a-16 c_{0}=256\{(3,0)+(2,2) \\
+(1,1)-(2,0)\} \equiv 0(\bmod 256) .
\end{gathered}
$$

By (12), $8 a$ can be replaced by $8 x-32 b$ above. Then (15) follows, with use of (10) and (14).

Next suppose that $4 \| m$. We must show that

$$
c_{0} \equiv 2 b+7(\bmod 16) \text {. }
$$

Assume that $2 \mid f$. Then

$$
\begin{gathered}
2 p+2+4 x+8 a+64 y+16 c_{0}=256\{(3,11)+(1,8) \\
+(2,8)-(2,10)\} \equiv 0(\bmod 256) .
\end{gathered}
$$

By (12), $8 a$ may be replaced by $8 x-32 b$ above. Then (16) follows, with use of (10) and (14). Now assume that $2 \nmid f$. Then

$$
2 p+2+4 x-24 a-16 c_{0}=256\{(3,5)+(2,1)\} \equiv 0(\bmod 256) .
$$

By (12), $24 a$ may be replaced by $24 x-96 b$ above. Then (16) follows, with use of (14).

Finally, suppose that $2 \| m$. Then $2 \| c_{2}$ by (11). Since by (8), $c_{0} \equiv-1(\bmod 8)$ and

$$
16 f+1=p \equiv c_{0}^{2}+2 c_{2}^{2}+2\left(c_{1}^{2}+c_{3}^{2}\right)(\bmod 32),
$$

the result follows.

We now give an elementary proof of (9), the congruence $y \equiv$ $2 b+m(\bmod 16)$. Our proof will use the useful and easily proved 
fact $[6, p .426]$ that the cyclotomic number $(0, j)$ is odd if and only if $j \equiv m(\bmod 16)$.

THEOREM 3. We have $y \equiv 2 b+m(\bmod 16)$.

Proof. Whiteman [11, p. 411] has given an elementary proof in the case $8 \mid \mathrm{m}$. It remains to consider the cases $2 \| m$ and $4 \| m$. There is no loss of generality in assuming that $m \equiv 2$ or $m \equiv 4$ $(\bmod 16)$.

Case $1 . \quad m \equiv 2(\bmod 16), 2 \mid f$.

Since $(0,2)$ is odd,

$$
\begin{aligned}
4(p+1)-8 x & +64 b-32 y=256\{(0,2)-(0,6)+2(4,8) \\
& +2(4,10)\} \equiv 256(\bmod 512) .
\end{aligned}
$$

By (14), $8 x$ can be replaced by $212-12 p$ above, and the result follows.

Case 2. $m \equiv 2(\bmod 16), 2 \nmid f$.

Since $(0,2)$ is odd,

$$
\begin{gathered}
8 x+28-4 p-32 y-32 a-64 b=256\{(0,2)-(0,6) \\
-2(4,2)-2(4,0)\} \equiv 256(\bmod 512) .
\end{gathered}
$$

Using (13) and (14), we deduce that $y+2 b \equiv 10(\bmod 16)$. Since $2 \| b$ by (11), it follows that $y \equiv 2 b+2(\bmod 16)$.

Case 3. $m \equiv 4(\bmod 16), 2 \mid f$.

Since $(0,4)$ is odd,

$$
32 c_{0}+32 y-14 x-p-17=256\{(0,4)-2(2,10)\} \equiv 256(\bmod 512) .
$$

By Theorem $1,2 x \equiv 9 p-71(\bmod 512)$, and by Theorem $2, c_{0} \equiv 2 b+$ $7(\bmod 16)$. We thus deduce that $y \equiv 2 b+4(\bmod 16)$.

Case $4 . \quad m \equiv 4(\bmod 16), 2 \nmid f$.

Since $(0,4)$ is odd,

$$
\begin{gathered}
2 p-62-4 x+32 y-32 a+32 c_{0}=256\{(0,0)-(0,4) \\
+2(2,0)\} \equiv 256(\bmod 512) .
\end{gathered}
$$

By Theorem $1,2 x \equiv p-127(\bmod 512)$, and by Theorem $2, c_{0} \equiv 2 b+$ 
$7(\bmod 16)$. By $(13), 2 a \equiv 3-p(\bmod 32) . \quad$ We thus deduce that $y \equiv 2 b+4(\bmod 16)$.

In the next theorem, we characterize $c_{2}(\bmod 8)$, thus extending (11).

THEOREM 4. We have

$$
c_{2} \equiv \begin{cases}b(\bmod 8), & \text { if } 8|m, 2| f \text { or } 4 \| m, 2 \nmid f \\ (-1)^{f+(m-2) / 4} b(\bmod 8), & \text { if } 2 \| m \\ b+4(\bmod 8), & \text { if } 4 \| m, 2 \mid f \text { or } 8 \mid m, 2 \nmid f .\end{cases}
$$

Proof. First suppose that $2 \| m$. It suffices to consider the case $m \equiv 2(\bmod 8)$. When $2 \mid f$,

$$
256\{(2,4)-(4,10)\}=32\left(c_{2}-b\right),
$$

and so $c_{2} \equiv b(\bmod 8)$. When $2 \nmid f$,

$$
256\{(4,2)-(2,6)\}=32\left(b+c_{2}\right),
$$

and so $c_{2} \equiv-b(\bmod 8)$.

Finally, suppose that $4 \mid \mathrm{m}$. Cyclotomic numbers are not needed in this case. By (11), $4 \mid b$ and $4 \mid c_{2}$. By Theorem 2, $c_{0} \equiv 2(b+m)$ $-1(\bmod 16)$, so $c_{0}^{2} \equiv 4(b+m)(b+m-1)+1(\bmod 32)$. Hence, by $(8)$,

$$
p-1=16 f \equiv 4(b+m)(b+m-1)+2\left(c_{1}^{2}+c_{3}^{2}\right)(\bmod 32) .
$$

Thus,

$$
\left\{\begin{array}{l}
4\left|c_{1}, 4\right| c_{3}, \text { if } 4 f \equiv b+m(\bmod 8) \\
2|| c_{1}, 2 \| c_{3}, \text { if } 4 f \not \equiv b+m(\bmod 8) .
\end{array}\right.
$$

Also by (8), $2 c_{0} c_{2}=c_{1}^{2}-c_{3}^{2}-2 c_{1} c_{3}$, so

$$
\left\{\begin{array}{l}
8 \mid c_{2}, \text { if } 4 f \equiv b+m(\bmod 8) \\
4 \| c_{2}, \text { if } 4 f \neq \equiv b+m(\bmod 8) .
\end{array}\right.
$$

We thus obtain the result.

Incidentally, Theorem 4 and (11) yield the following criterion for the octic character of 2 modulo primes $p=16 f+1$ :

2 is an octic residue $(\bmod p)$ iff $4 \mid b$ and $b \equiv c_{2}+4 f(\bmod 8)$. Of course, simpler criteria (in terms of $y$ ) are known [10].

3. Resolution of sign ambiguities. We begin with quartic 
Jacobi and Jacobsthal sums in the case $4 \| m$, i.e., 2 is a quartic but not octic residue $(\bmod p)$. Without loss of generality, let $m \equiv$ $4(\bmod 16)$. Note that $4 \mid b$, by $(11)$.

THEOREM 5. Let $\psi$ be a character $(\bmod p)$ of order 4 chosen such that $\psi(g)=i$. Suppose that $m \equiv 4(\bmod 16)$. When $p \nmid \alpha$, define $r=\operatorname{ind}_{g} \alpha . \quad$ Then

$$
K(\psi)=-x+2 i y
$$

and

$$
\varphi_{2}(\alpha)= \begin{cases}-(-1)^{r / 2} 2 x, & \text { if } 2 \mid r \\ (-1)^{(r-1) / 2} 4 y, & \text { if } 2 \nmid r\end{cases}
$$

where $x$ and $y$ are uniquely determined by the conditions

$$
p=x^{2}+4 y^{2}, x \equiv 1(\bmod 4), \text { and } y \equiv\left\{\begin{array}{l}
4(\bmod 16), \text { if } 8 \mid b \\
-4(\bmod 16), \text { if } 4 \| b .
\end{array}\right.
$$

Proof. The evaluation of $K(\psi)$ follows from (2), (6), and Theorem 3. The evaluation of $\varphi_{2}(\alpha)$ then follows with use of (1).

For quartic sums $K(\psi)$ and $\varphi_{2}(\alpha)$ modulo primes $\equiv 1(\bmod 4)$ for which 2 is not a quartic residue, the sign ambiguities have been resolved (in terms of $m$ ) by E. Lehmer [7], [8]. The signs remain undetermined for $K(\psi)$ and $\varphi_{2}(\alpha)$ modulo primes $\equiv 1(\bmod 16)$ for which 2 is an octic residue, and also modulo primes $\equiv 9(\bmod 16)$ for which 2 is a quartic residue.

An evaluation of $\varphi_{6}(\alpha)$ modulo primes $\equiv 1(\bmod 12)$, up to some undetermined signs, is given in [1, Theorem 4.8]. However, for the particular primes $\equiv 1(\bmod 12)$ for which 2 is not a quartic residue and the primes $\equiv 1(\bmod 48)$ for which 2 is a quartic but not octic residue, $\varphi_{6}(\alpha)$ can be completely determined. This is because $K(\psi)$ is completely determined for these primes, and consequently so is $\varphi_{6}(\alpha)$ by [1, eqs. (4.3) and (4.5)].

We now consider octic and bioctic Jacobi and Jacobsthal sums in the case $2 \| m$. Without loss of generality, let $m \equiv 2(\bmod 16)$. The signs of $y$ and $b$ are simply determined, since $y \equiv-2(\bmod 8)$ by $(10)$ and $b \equiv-1+y / 2(\bmod 8)$ by Theorem 3 .

THEOREM 6. Let $\lambda$ be a character $(\bmod p)$ of order 8 chosen such that $\lambda(g)=e^{2 \pi i / 8}$. Suppose that $m \equiv 2(\bmod 16)$. When $p \nmid \alpha$, define $r=\operatorname{ind}_{g} \alpha$. Then

$$
K(\lambda)=-a+i b \sqrt{2}
$$


and

$$
\varphi_{4}(\alpha)=\left\{\begin{array}{cl}
-(-1)^{r / 4} 4 a, & \text { if } 4 \mid r \\
0 & , \text { if } 2 \| r \\
4 b & , \text { if } r \equiv 1 \text { or } r \equiv 3(\bmod 8) \\
-4 b & , \text { if } r \equiv 5 \text { or } r \equiv 7(\bmod 8),
\end{array}\right.
$$

where $a$ and $b$ are uniquely determined by the conditions

$$
\begin{aligned}
p=a^{2}+2 b^{2}, a & \equiv 1(\bmod 4), \\
\text { and } b & \equiv\left\{\begin{array}{c}
2(\bmod 8), \text { if } y \equiv 6(\bmod 16) \\
-2(\bmod 8), \text { if } y \equiv-2(\bmod 16) .
\end{array}\right.
\end{aligned}
$$

Proof. The evaluation of $K(\lambda)$ follows from (3), (7), and Theorem 3. The evaluation of $\varphi_{4}(\alpha)$ then follows with use of (1).

The octic sums $K(\lambda)$ and $\varphi_{4}(\alpha)$ that remain ambiguous are those modulo primes $\equiv 9(\bmod 16)$, and modulo primes $\equiv 1(\bmod 16)$ for which 2 is a quartic residue.

THEOREM 7. Let $\chi$ be a character $(\bmod p)$ of order 16 chosen such that $\chi(g)=e^{2 \pi i / 16}$. Suppose that $m \equiv 2(\bmod 16)$. When $p \nmid \alpha$, define $r=\operatorname{ind}_{g} \alpha$. Then

$$
K(\chi)=c_{0}+c_{2} \sqrt{2}+i c_{1} \sqrt{2-\sqrt{2}}+i c_{3} \sqrt{2+\sqrt{2}}
$$

and

$$
(-1)^{f} \varphi_{8}(\alpha)=\left\{\begin{aligned}
(-1)^{r / 8} 8 c_{0}, & \text { if } 8 \mid r \\
0 & , \text { if } 4 \| r \\
8 c_{2} \text { or } & -8 c_{2} \text { according as } r \equiv \pm 2 \text { or } \pm 6(\bmod 16) \\
8 c_{1} & , \text { if } r \equiv 1 \text { or } r \equiv 7(\bmod 16) \\
-8 c_{1} & , \text { if } r \equiv 9 \text { or } r \equiv 15(\bmod 16) \\
8 c_{3} & , \text { if } r \equiv 3 \text { or } r \equiv 5(\bmod 16) \\
-8 c_{3} & , \text { if } r \equiv 11 \text { or } r \equiv 13(\bmod 16)
\end{aligned}\right.
$$

where $c_{0}$ and $c_{2}$ are uniquely determined by the conditions

$$
p=c_{0}^{2}+2 c_{1}^{2}+2 c_{2}^{2}+2 c_{3}^{2}, c_{0} \equiv-1(\bmod 8), 2 c_{0} c_{2}=c_{1}^{2}-c_{3}^{2}-2 c_{1} c_{3} \text {, and }
$$

$$
(-1)^{f} c_{2} \equiv\left\{\begin{array}{c}
2(\bmod 8), \text { if } y \equiv 6(\bmod 16) \\
-2(\bmod 8) \text {, if } y \equiv-2(\bmod 16) .
\end{array}\right.
$$

Proof. The evaluation of $K(\chi)$ follows from (4), (8), and Theorems 3 and 4 . The evaluation of $\varphi_{8}(\alpha)$ then follows with the aid 
of (1) (see [2, Theorem 3.9]).

Theorem 7 gives only a partial resolution of signs, because while $c_{0}$ and $c_{2}$ are determined, $c_{1}$ and $c_{3}$ are not. Theorem 8 below shows that $c_{1}$ and $c_{3}$ can also be determined in Theorem 7 if one makes the additional assumption that $2 \mid f$, i.e., $p \equiv 1(\bmod 32)$. We assume without loss of generality that $m \equiv 2(\bmod 32)$.

THEOREM 8. If in Theorem 7 the additional assumptions $2 \mid f$ and $m \equiv 2(\bmod 32)$ are made, then the evaluations of $K(\chi)$ and $\varphi_{8}(\alpha)$ are valid with $c_{0}, c_{1}, c_{2}$, and $c_{3}$ uniquely determined by the conditions in (17) together with the conditions

$$
\left\{\begin{array}{l}
4 \mid c_{1} \text { and } 2 \| c_{3}, \text { if } y \equiv 6(\bmod 16) \\
4 \mid c_{3} \text { and } 2 \| c_{1}, \text { if } y \equiv-2(\bmod 16)
\end{array}\right.
$$

and

$$
c_{1}+c_{3} \equiv(y+2 b-2) / 4(\bmod 8)
$$

Proof. The conditions in (18) are easily proved with use of (17). Hasse $[5$, p. 233] proved that when $p \equiv 1(\bmod 32)$,

$$
y+2 b-4\left(c_{1}+c_{3}\right) \equiv m(\bmod 32) .
$$

Thus (19) follows from (20). To see that the signs of $c_{1}$ and $c_{3}$ are uniquely determined by (17), (18), and (19), note first that $c_{1}+c_{3} \equiv$ $\pm 2(\bmod 8)$ by $(18)$, so that the sign of $c_{1}+c_{3}$ is determined by (19). The result now follows because the sign of $2 c_{1} c_{3}=c_{1}^{2}-c_{3}^{2}-2 c_{0} c_{2}$ is determined by (17) and (18).

The bioctic sums $K(\chi)$ and $\varphi_{8}(\alpha)(\bmod p)$ that remain ambiguous are those for which $p \equiv 17(\bmod 32)$, and those for which $p \equiv 1$ $(\bmod 32)$ with 2 a quartic residue $(\bmod p)$.

Note added in proof. Congruences (9) and (20) are the cases $n=4$ and $n=5$ of a general congruence $\left(\bmod 2^{n}\right)$ conjectured by J. B. Muskat in 1971. The author has recently obtained an elementary proof of Muskat's conjecture.

\section{REFERENCES}

1. B. C. Berndt and R. J. Evans, Sums of Gauss, Jacobi, and Jacobsthal, J. Number Theory, (to appear, 1979).

2. - Sums of Gauss, Eisenstein, Jacobi, Jacobsthal, and Brewer, Illinois J. Math., (to appear, 1979). 
3. R. J. Evans, The cyclotomic numbers of order sixteen, Math. Comp., 33 (1979), 827-835.

4. R. E. Giudici, J. B. Muskat, and S. F. Robinson, On the evaluation of Brewer's character sums, Trans. Amer. Math. Soc., 171 (1972), 317-347.

5. H. Hasse, Der $2^{n}-t e$ Potenzcharakter von 2 im Körper der $2^{n}$-ten Einheitswurzeln, Rend, Circ. Mat. di Palermo, Serie II, 7 (1958), 185-243.

6. E. Lehmer, On residue difference sets, Canad. J. Math., 5 (1953), 425-432.

7. - On the number of solutions of $u^{k}+D \equiv w^{2}(\bmod p)$, Pacific J. Math., 5 (1955), 103-118.

8. - On Jacobi functions, Pacific J. Math., 10 (1960), 887-893.

9. J. B. Muskat and Y. C. Zee, On the uniqueness of solutions of certain Diophantine equations, Proc. Amer. Math. Soc., 49 (1975), 13-19.

10. A. L. Whiteman, The sixteenth power residue character of 2, Canad. J. Math., 6 (1954), 364-373.

11.

The cyclotomic numbers of order sixteen, Trans. Amer. Math. Soc., 86 (1957), 401-413.

Received April 5, 1978, and in revised form July 10, 1978.

University of California, San Diego

LA JOLla, CA 92093 


\section{PACIFIC JOURNAL OF MATHEMATICS}

EDITORS

DONALD BABBITT (Managing Editor)

University of California

Los Angeles, California 90024

HUGo RossI

University of Utah

Salt Lake City, UT 84112

C. C. MOORE

J. DUGUNDJI

Department of Mathematics

University of Southern California

Los Angeles, California 90007

R. FinN AND J. MiLgraM

Stanford University

Stanford, California 94305

University of California

Berkeley, CA 94720

\section{ASSOCIATE EDITORS}

E. F. BrCKENBACH

B. H. NeUmanN

F. WOLF

K. YoshidA

\section{SUPPORTING INSTITUTIONS}

UNIVERSITY OF BRITISH COLUMBIA

UNIVERSITY OF SOUTHERN CALIFORNIA

CALIFORNIA INSTITUTE OF TECHNOLOGY

STANFORD UNIVERSITY

UNIVERSITY OF CALIFORNIA

UNIVERSITY OF HAWAII

MONTANA STATE UNIVERSITY

UNIVERSITY OF TOKYO

UNIVERSITY OF NEVADA, RENO

UNIVERSITY OF UTAH

NEW MEXICO STATE UNIVERSITY

WASHINGTON STATE UNIVERSITY

OREGON STATE UNIVERSITY

UNIVERSITY OF WASHINGTON

UNIVERSITY OF OREGON 


\section{Pacific Journal of Mathematics}

\section{Vol. 81, No. $1 \quad$ November, 1979}

Thomas E. Armstrong, Simplicial subdivision of infinite-dimensional compact cubes ..................................... 1

Herbert Stanley Bear, Jr., Approximate identities and pointwise convergence ................................

Richard David Bourgin, Partial orderings for integral representations on convex sets with the Radon-Nikodým property..................

Alan Day, Herbert S. Gaskill and Werner Poguntke, Distributive lattices

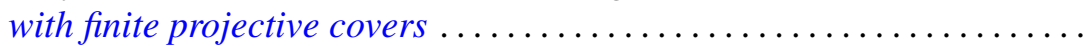

Heneri Amos Murima Dzinotyiweyi and Gerard L. G. Sleijpen, A note on measures on foundation semigroups with weakly compact orbits ......

Ronald James Evans, Resolution of sign ambiguities in Jacobi and Jacobsthal sums ...................................

John Albert Fridy, Tauberian theorems via block dominated matrices ......

Matthew Gould and Helen H. James, Automorphism groups retracting onto

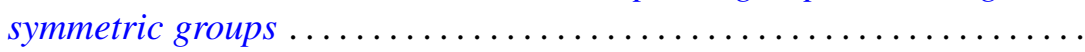

Kurt Kreith, Nonlinear differential equations with monotone solutions . . . . 101

Brian William McEnnis, Shifts on indefinite inner product spaces........ 113

Joseph B. Miles, On entire functions of infinite order with radially

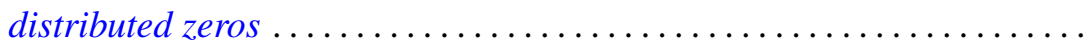

Janet E. Mills, The idempotents of a class of 0-simple inverse semigroups ...............................

Edward Jean Moulis, Jr., Generalizations of the Robertson functions ...

Richard A. Moynihan and Berthold Schweizer, Betweenness relations in probabilistic metric spaces.......................

Stanley Ocken, Perturbing embeddings in codimension two ....

Masilamani Sambandham, On the average number of real zeros of a class of random algebraic curves.

Jerry Searcy and B. Andreas Troesch, A cyclic inequality and a related eigenvalue problem.

Roger R. Smith and Joseph Dinneen Ward, $M$-ideals in $B\left(l_{p}\right)$...

Michel Talagrand, Deux généralisations d'un théorème de I. Namioka ..

Jürgen Voigt, $O n Y$-closed subspaces of $X$, for Banach spaces $X \subset Y$;

existence of alternating elements in subspaces of $C(J)$

Sidney Martin Webster, On mapping an $n$-ball into an $(n+1)$-ball in complex spaces

David J. Winter, Triangulable subalgebras of Lie p-algebras ... 